\title{
Survival benefit of perioperative chemotherapy for T1-3NOMO stage esophageal cancer: a SEER database analysis
}

\author{
Yue Shao $^{1 \#}$, Dan Chen ${ }^{1 \#}$, Liu Ye ${ }^{2}$, Xin-Mei Wang ${ }^{3}$, Qing-Chen $W^{1}{ }^{1}$, Cheng Zhang ${ }^{1}$ \\ ${ }^{1}$ Department of Cardiothoracic Surgery, The First Affiliated Hospital of Chongqing Medical University, Chongqing, China; ${ }^{2}$ The First Branch, \\ The First Affiliated Hospital of Chongqing Medical University, Chongqing, China; ${ }^{3}$ Department of Pathology, Chongqing Medical University, \\ Chongqing, China \\ Contributions: (I) Conception and design: C Zhang, Y Shao; (II) Administrative support: QC Wu; (III) Provision of study materials or patients: Y \\ Shao, D Chen; (IV) Collection and assembly of data: Y Shao, XM Wang; (V) Data analysis and interpretation: Y Shao, L Ye; (VI) Manuscript writing: \\ All authors; (VII) Final approval of manuscript: All authors. \\ "These authors contributed equally to this work and should be considered as co-first authors. \\ Correspondence to: Cheng Zhang. Department of Cardiothoracic Surgery, The First Affiliated Hospital of Chongqing Medical University, No. 1 Youyi \\ Road, Yuzhong District, Chongqing, China. Email: zhangchengcqmu@126.com.
}

\begin{abstract}
Background: The effect of perioperative chemotherapy on patients with lymph node-negative esophageal cancer (EC) is controversial. This study explored which EC patients, staged under the T1-3N0M0, would benefit from perioperative chemotherapy.

Methods: Data on patients with diagnosed primary EC were retrieved from Surveillance, Epidemiology and End Results (SEER) database. Propensity score-matched (PSM) method was performed to balance baseline covariates. Multivariate Cox regression analysis and Kaplan-Meier curve were used to assess potential survival difference between patients undergoing surgery plus perioperative chemotherapy (SA + CT) and those undergoing surgery alone (SA).

Results: In a total of 2,711 EC patients (T1-3N0M0), 166 patients underwent SA + CT and 2,545 patients received SA. In the multivariable analysis, $\mathrm{T}$ stage was significantly related to prognosis of EC patients before and after matching. Subgroup analysis showed that perioperative chemotherapy was associated with poor cancer-specific survival (CSS) for stage T1 patients. There was no effect of perioperative chemotherapy on overall survival (OS) or CSS for T2 patients, whereas a remarkable improvement in OS and CSS was observed for T3 patients. Survival analysis showed that T3 stage EC patients obtained survival benefit from SA + CT. Prognosis in the SA group was significantly better than in the SA + CT group for T1 patients. However, T2 patients showed no significant increase in survival after undergoing SA + CT compared with SA.

Conclusions: T3 patients benefit more from SA + CT. However, perioperative chemotherapy does not present survival benefit to T1-2 patients, and it is an adverse prognostic factor for T1 patients.
\end{abstract}

Keywords: Esophageal cancer (EC); perioperative chemotherapy; cancer-specific survival (CSS); overall survival (OS)

Submitted Sep 13, 2020. Accepted for publication Dec 17, 2020.

doi: $10.21037 /$ jtd-20-2877

View this article at: http://dx.doi.org/10.21037/jtd-20-2877

\section{Introduction}

Esophageal cancer (EC) is a common malignant digestive tract tumor and the sixth most common cause of cancerrelated death worldwide $(1,2)$. Esophageal adenocarcinoma (EAC) and esophageal squamous cell carcinoma (ESCC) are the two main histological subtypes of EC (3). Currently, chemotherapy, radiotherapy and surgery are the conventional treatment strategies for different types of cancers. Surgery is recommended for treatment of earlystage EC, whereas adjuvant chemoradiotherapy is preferred 
for local advanced EC. The five-year overall survival (OS) rate of EC is only $15-25 \%$ despite efforts to improve the diagnosis and therapy $(4,5)$.

Theoretically, adjuvant chemotherapy can effectively reduce the recurrence rate and prolong survival by eliminating the potential residual tumor cell (6). Previous clinical trials report that adjuvant therapy can significantly improve survival for patients with positive pathological lymph nodes $(7,8)$. However, it is controversial whether adjuvant therapy is beneficial to the survival of pathologically node-negative EC.

The aim of this study was to compare the OS and cancer-specific survival (CSS) of EC patients who underwent surgery alone (SA) or surgery plus perioperative chemotherapy (SA + CT) based on population-based data from the SEER database, after performing PSM. We present the following article in accordance with the STROBE reporting checklist (available at http://dx.doi. org/10.21037/jtd-20-2877).

\section{Methods}

\section{Study population}

All data used in this study was derived from the SEER database, which is an open-access webserver (https://seer. cancer.gov/). The SEER database is an authoritative source of clinical information, demographic data, cancer incidence, and survival, which covers 18 cancer registries and represents nearly $28 \%$ of the US population. The study was conducted in accordance with the Declaration of Helsinki (as revised in 2013). Requirement for informed consent was waived since the SEER database is publicly available and anonymous. This study was approved by the Ethics Committee of the First Affiliated Hospital of Chongqing Medical University, Chongqing, China as exempted research with no human subject involved.

SEER*Stat software (version 8.3.6) was used to select the study population. All primary cases of EC were identified (International Classification of Diseases for Oncology (ICD-O-3) anatomic codes 150-155 and 158-159). Information on age, sex, race, tumor size, nuclear grade, location, histological type, number of examined lymph node (ELN), treatment, T stage, and survival was collected for each case from the database. Histologic codes of EAC and ESCC were 8140-8389 and 8050-8089 respectively. In this study, upper esophagus codes were C15.0 and C15.3. Middle esophagus was defined as Code C15.4. A combination of C15.2 and C15.5 was used to represent the lower esophagus. The OS and CSS were primary endpoints. The OS was defined as the time interval between initial diagnosis and death from any cause. The CSS referred to the interval from initial diagnosis to the occurrence of ECspecific death. Tumor stage was identified based on the sixth edition tumor-node-metastasis (TNM) staging of the American Joint Committee on Cancer (AJCC).

Patient inclusion and exclusion criteria are shown in Figure 1. Inclusion criteria were: (I) patients with primary EC aged $>18$ years between January 2004 and December 2016; (II) patients with T1-3N0M0 stage EC who underwent surgical resection. Exclusion criteria included: (I) patients whose survival time was missing or was 0; (II) patients who did not undergo surgery or unknown local treatment; (III) patients who received radiotherapy; (IV) patients diagnosed with $\mathrm{T} 4$ or Tis status, positive lymph node, unknown or positive metastatic status.

\section{Statistical analysis}

Patients were divided into two groups based on presence or absence of perioperative chemotherapy. Comparison of categorical variables between the two groups was performed using the Pearson chi-square test and expressed as counts and percentages. To minimize the effects of potential bias, a 1:1 nearest-neighbor PSM method was performed using a 0.05 standard deviation caliper width on the R package "MatchIt" (https://cran.r-project.org/web/packages/MatchIt/index). Matching variables included age, gender, race, pathologic grade, T stage, size of tumor, location, histological type, and ELN count. Multivariable Cox proportional hazards models were constructed to explore survival-related factors, and the hazard ratio (HR) with a $95 \%$ confidence interval (CI) for each variable was calculated.

An interaction test was conducted to explore whether the subgroups of different $T$ stages had any survival benefit after perioperative chemotherapy. Residual factors were adjusted in the multivariable Cox regression model when examining the benefit of perioperative chemotherapy in patients stratified using the AJCC T stage. These factors included the age of patients, race, gender, nuclear grade, histology, ELN count, location, and tumor size. The Kaplan-Meier method was constructed to evaluate survival curves of patients before and after matching and results were compared by using log-rank tests. In addition, another three pairs of survival curves were plotted based on different T stages subgroups. $\mathrm{P}<0.05$ was considered to be statistically 


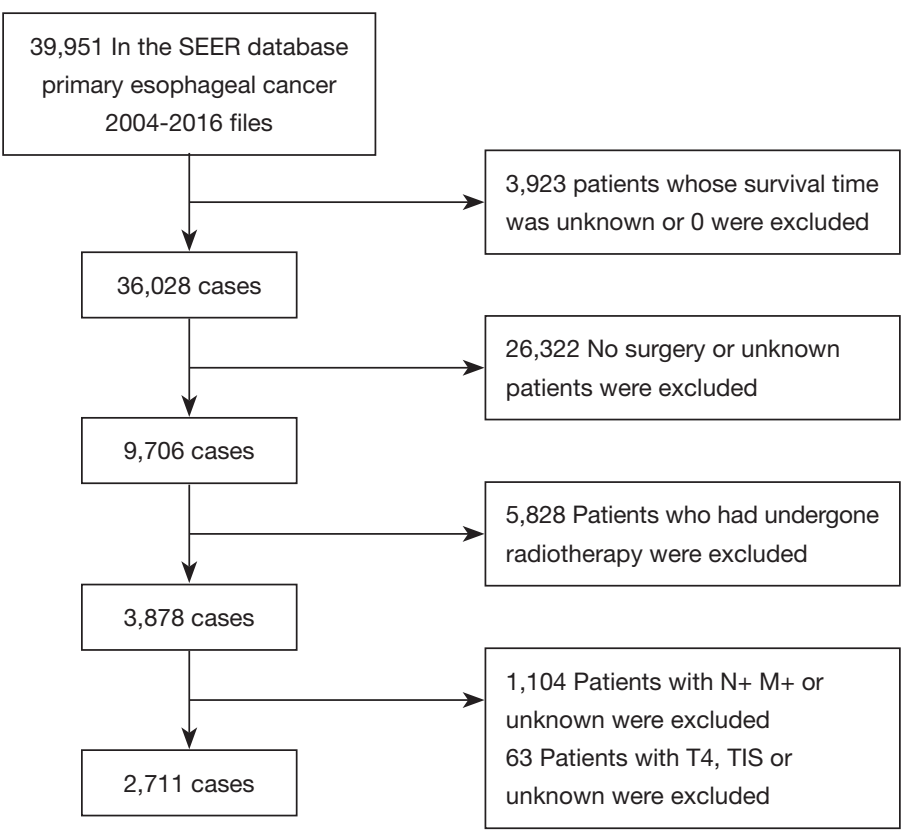

Figure 1 The flow diagram of the selection process for the study.

significant. All statistical analyses were performed using the R software (version 3.6.2, http://www.r-project.org/).

\section{Results}

\section{Patient characteristics}

We selected 2,711 cases from the SEER database following the inclusion and exclusion criteria. A total of $2,545(93.88 \%)$ cases received SA, whereas $166(6.12 \%)$ cases received $\mathrm{SA}+\mathrm{CT}$. The clinical and demographic characteristics of SA and $\mathrm{SA}+\mathrm{CT}$ groups are shown in Table 1. Age, grade, ELN count, T stage, histology, location, and tumor size showed significant differences between SA and SA + CT groups before matching. Specifically, the SA + CT group showed higher proportions of younger patients ( $<65$ years), higher differential grade, more ELN count, higher T stage, less adenocarcinoma, lower location, more tumor size $>30 \mathrm{~mm}$ compared with the SA group (all $\mathrm{P}<0.05$ ). Baseline characteristics of patients in the two groups were balanced after PSM. Residual characteristics were similarly distributed between the two groups (all $\mathrm{P}>0.05)$, except for $\mathrm{ELN}$ count $(\mathrm{P}=0.013)$.

\section{Survival analysis}

The mean follow-up period was 46 months. Survival curves of SA + CT group versus SA group, before and after matching, are shown in Figure 2. Before matching, the 5 -year OS rate of the SA group was $66.1 \%$, whereas the 5 -year OS rate of the SA + CT group was 53\% (log-rank $\mathrm{P}=0.0011$; Figure 2A). Similarly, Patients who underwent SA showed better CSS compared with patients in the SA + CT group (log-rank $\mathrm{P}<0.0001$; Figure $2 B$ ). However, no significant survival differences were detected between the two groups (log-rank $\mathrm{P}=0.39$ for OS and $\mathrm{P}=0.55$ for CSS; Figure $2 C, D)$, considering all matched patients.

The cohort, before and after matching, was analyzed using multi-factors (Table 2). Compared to the SA group, the SA + CT group displayed no differences in the OS (HR $=0.86 ; 95 \% \mathrm{CI}, 0.67-1.10 ; \mathrm{P}=0.232)$ and $\mathrm{CSS}(\mathrm{HR}=0.84$; 95\% CI, 0.61-1.14; $\mathrm{P}=0.264)$ in the original Cox models. The propensity score-matched (PSM) Cox regression model showed similar results. Before matching, T1 patients had significantly better prognosis compared with $\mathrm{T} 2$ and T3 patients $(\mathrm{P}<0.001)$. After matching, only patients at $\mathrm{T} 3$ stage showed significant differences in OS ( $\mathrm{HR}=2 ; 95 \%$ CI, 1.29-3.09; $\mathrm{P}=0.002)$ and $\mathrm{CSS}(\mathrm{HR}=2.13 ; 95 \% \mathrm{CI}$, 1.25-3.62; $\mathrm{P}=0.006)$, where the patients at $\mathrm{T} 1$ stage were taken as the reference. Also, in the unmatched cohort, age, grade, ELN number, pathological type, and tumor location were related to OS and CSS, but these factors showed no correlation with prognosis in the matched cohort. 
Table 1 Comparison of baseline variables between surgery alone and surgery plus perioperative chemotherapy groups in the original and matched datasets in cases of esophageal cancer

\begin{tabular}{|c|c|c|c|c|c|c|}
\hline Characteristic & \multicolumn{3}{|c|}{ Original data set } & \multicolumn{3}{|c|}{ Matched data set } \\
\hline Total & 2545 & 166 & & 148 & 148 & \\
\hline Race & & & 1.000 & & & 0.574 \\
\hline White & 2,325 (91.4) & $152(91.6)$ & & $130(87.8)$ & $134(90.5)$ & \\
\hline Female & $458(18.0)$ & $24(14.5)$ & & $17(11.5)$ & $24(16.2)$ & \\
\hline Male & 2,087 (82.0) & $142(85.5)$ & & $131(88.5)$ & $124(83.8)$ & \\
\hline Age & & & 0.002 & & & 0.907 \\
\hline G1-2 & $1,423(55.9)$ & $71(42.8)$ & & $77(52.0)$ & $66(44.6)$ & \\
\hline G3-4 & $537(21.1)$ & $74(44.6)$ & & $50(33.8)$ & 65 (43.9) & \\
\hline Unknown & $585(23.0)$ & $21(12.7)$ & & $21(14.2)$ & $17(11.5)$ & \\
\hline ELN count & & & $<0.001$ & & & 0.013 \\
\hline $0-3$ & $1,143(44.9)$ & 23 (13.9) & & $22(14.9)$ & $23(15.5)$ & \\
\hline$\geq 4$ & $1,337(52.5)$ & 141 (84.9) & & $115(77.7)$ & $124(83.8)$ & \\
\hline Unknown & $65(2.6)$ & $2(1.2)$ & & $11(7.4)$ & $1(0.7)$ & \\
\hline T stage & & & $<0.001$ & & & 0.389 \\
\hline SCC & $383(15.0)$ & $31(18.7)$ & & $42(28.4)$ & $31(20.9)$ & \\
\hline Other & $162(6.4)$ & $20(12.0)$ & & $11(7.4)$ & $16(10.8)$ & \\
\hline Tumor location & & & 0.022 & & & 0.057 \\
\hline Lower & 1,837 (72.2) & $128(77.1)$ & & $115(77.7)$ & $112(75.7)$ & \\
\hline Middle & 328 (12.9) & $9(5.4)$ & & 17 (11.5) & $8(5.4)$ & \\
\hline Upper & $69(2.7)$ & $5(3.0)$ & & $5(3.4)$ & $5(3.4)$ & \\
\hline Unknown & $311(12.2)$ & $24(14.5)$ & & $11(7.4)$ & $23(15.5)$ & \\
\hline Tumor size & & & $<0.001$ & & & 0.357 \\
\hline$\leq 30 \mathrm{~mm}$ & $1,418(55.7)$ & 57 (34.3) & & $63(42.6)$ & $54(36.5)$ & \\
\hline$>30 \mathrm{~mm}$ & 367 (14.4) & $73(44.0)$ & & $53(35.8)$ & 65 (43.9) & \\
\hline Unknown & 760 (29.9) & $36(21.7)$ & & 32 (21.6) & $29(19.6)$ & \\
\hline
\end{tabular}

$\mathrm{SA}$, surgery alone; SA + CT, surgery plus perioperative chemotherapy; ELN, examined lymph node; SCC, squamous cell carcinoma; AC, adenocarcinoma. 

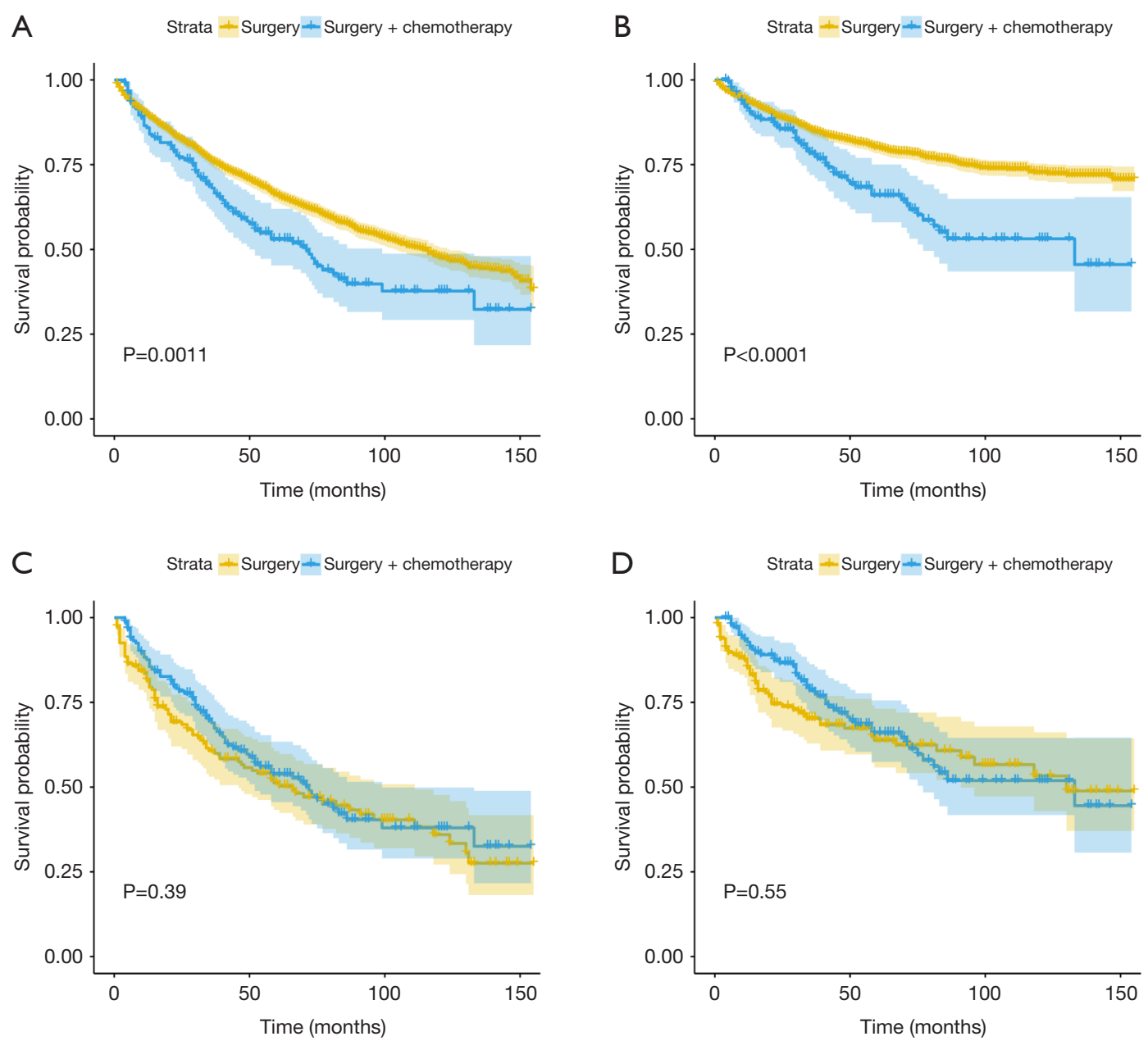

Figure 2 Survival comparisons between surgery alone and surgery + perioperative chemotherapy groups. Overall survival comparisons between patients with surgery alone and surgery + perioperative chemotherapy in whole cohort (A) and matched cohort (C). Cause-specific survival comparisons between patients with surgery alone and surgery + perioperative chemotherapy in whole cohort (B) and matched cohort (D).

Interestingly, the cohorts showed that tumor size $>30 \mathrm{~mm}$ was a risk factor for CSS before and after PSM, but it was not significant for OS. In subgroup analysis (Table 3), T3 patients undergoing SA + CT had significantly better OS and CSS than those undergoing SA. Also, the CSS of the SA group was significantly better than the SA + CT group for $\mathrm{T} 1$ stage patients $(\mathrm{HR}=2.82$; 95\% CI, 1.04-7.67; $\mathrm{P}=0.042$ ).

Further survival analysis was performed according to the T1, T2, and T3 stages after matching, to explore the survival differences between the SA + CT and SA groups at different $\mathrm{T}$ stages of $\mathrm{EC}$. T1 stage EC patients benefited from SA (Figure $3 A, \mathrm{P}=0.025$; Figure $3 B, \mathrm{P}=0.0051$ ), and there was no significant difference between the prognosis of T2 EC patients receiving SA and SA + CT (Figure 3C,D). However, the $\mathrm{T} 3$ stage patients benefited from $\mathrm{SA}+\mathrm{CT}$ (Figure $3 E, \mathrm{P}=0.0024$; Figure $3 F, \mathrm{P}=0.0051$ ).

\section{Discussion}

The purpose of our study was to explore the survival differences of T1-3N0 patients who underwent SA versus SA + CT, based on a large sample size and PSM method. Patients at stage T3 benefited more from SA + CT compared with T1 and T2 patients. The CSS of the SA group was significantly better than that of the SA + CT 
Table 2 Multivariate Cox regression analysis for OS and CSS in overall patient cohort

\begin{tabular}{|c|c|c|c|c|c|c|c|c|}
\hline \multirow{3}{*}{ Characteristic } & \multicolumn{4}{|c|}{ Unmatched cohort $(\mathrm{N}=2711)$} & \multicolumn{4}{|c|}{ Matched cohort $(\mathrm{N}=296)$} \\
\hline & \multicolumn{2}{|l|}{ OS } & \multicolumn{2}{|l|}{ CSS } & \multicolumn{2}{|l|}{ os } & \multicolumn{2}{|l|}{ CSS } \\
\hline & $\mathrm{HR}(95 \% \mathrm{Cl})$ & $\mathrm{P}$ & $\mathrm{HR}(95 \% \mathrm{Cl})$ & $\mathrm{P}$ & $\mathrm{HR}(95 \% \mathrm{Cl})$ & $\mathrm{P}$ & $\mathrm{HR}(95 \% \mathrm{Cl})$ & $\mathrm{P}$ \\
\hline White & $\mathrm{RF}$ & $\mathrm{RF}$ & $\mathrm{RF}$ & $\mathrm{RF}$ & $\mathrm{RF}$ & $\mathrm{RF}$ & $\mathrm{RF}$ & $\mathrm{RF}$ \\
\hline Other & $0.91(0.73-1.14)$ & 0.418 & $0.97(0.72-1.29)$ & 0.812 & $0.98(0.54-1.75)$ & 0.936 & $1.16(0.58-2.30)$ & 0.677 \\
\hline \multicolumn{9}{|l|}{ Treatment } \\
\hline SA & $\mathrm{RF}$ & $\mathrm{RF}$ & $\mathrm{RF}$ & $\mathrm{RF}$ & $\mathrm{RF}$ & $\mathrm{RF}$ & $\mathrm{RF}$ & $\mathrm{RF}$ \\
\hline $\mathrm{SA}+\mathrm{CT}$ & $0.86(0.67-1.10)$ & 0.232 & $0.84(0.61-1.14)$ & 0.264 & $0.78(0.56-1.11)$ & 0.165 & $0.78(0.51-1.19)$ & 0.251 \\
\hline \multicolumn{9}{|l|}{ Gender } \\
\hline Female & $\mathrm{RF}$ & $\mathrm{RF}$ & RF & $\mathrm{RF}$ & $\mathrm{RF}$ & $\mathrm{RF}$ & $\mathrm{RF}$ & $\mathrm{RF}$ \\
\hline$\geq 65$ & $1.79(1.57-2.04)$ & $<0.001$ & $1.52(1.27-1.82)$ & $<0.001$ & $1.01(0.72-1.41)$ & 0.961 & $0.92(0.61-1.39)$ & 0.680 \\
\hline \multicolumn{9}{|l|}{ Grade } \\
\hline G1-2 & $\mathrm{RF}$ & $\mathrm{RF}$ & $\mathrm{RF}$ & $\mathrm{RF}$ & $\mathrm{RF}$ & $\mathrm{RF}$ & $\mathrm{RF}$ & $\mathrm{RF}$ \\
\hline G3-4 & $1.28(1.11-1.48)$ & 0.001 & $1.62(1.34-1.96)$ & $<0.001$ & $1.41(0.99-2.01)$ & 0.057 & $1.44(0.93-2.21)$ & 0.100 \\
\hline Unknown & $0.85(0.71-1.03)$ & 0.102 & $0.69(0.50-0.94)$ & 0.017 & $1.07(0.54-2.09)$ & 0.850 & $0.91(0.38-2.19)$ & 0.842 \\
\hline \multicolumn{9}{|l|}{ ELN count } \\
\hline $0-3$ & $\mathrm{RF}$ & $\mathrm{RF}$ & $\mathrm{RF}$ & $\mathrm{RF}$ & $\mathrm{RF}$ & $\mathrm{RF}$ & $\mathrm{RF}$ & $\mathrm{RF}$ \\
\hline$\geq 4$ & $0.78(0.68-0.90)$ & $<0.001$ & $0.98(0.80-1.20)$ & 0.815 & $0.86(0.52-1.42)$ & 0.551 & $0.92(0.49-1.72)$ & 0.796 \\
\hline \multicolumn{9}{|l|}{ Histology } \\
\hline$A C$ & $\mathrm{RF}$ & $\mathrm{RF}$ & $\mathrm{RF}$ & $\mathrm{RF}$ & $\mathrm{RF}$ & $\mathrm{RF}$ & $\mathrm{RF}$ & $\mathrm{RF}$ \\
\hline SCC & $1.37(1.13-1.65)$ & 0.001 & $1.49(1.17-1.91)$ & 0.002 & $1.26(0.80-1.98)$ & 0.317 & $1.06(0.61-1.84)$ & 0.841 \\
\hline Other & $1.25(0.98-1.59)$ & 0.067 & $1.01(0.70-1.46)$ & 0.938 & $1.10(0.61-2.01)$ & 0.747 & $0.86(0.40-1.88)$ & 0.707 \\
\hline \multicolumn{9}{|l|}{ Tumor location } \\
\hline Lower & $\mathrm{RF}$ & $\mathrm{RF}$ & $\mathrm{RF}$ & $\mathrm{RF}$ & $\mathrm{RF}$ & $\mathrm{RF}$ & $\mathrm{RF}$ & $\mathrm{RF}$ \\
\hline Middle & $1.33(1.11-1.61)$ & 0.003 & $1.58(1.23-2.02)$ & $<0.001$ & $1.38(0.78-2.45)$ & 0.272 & $1.92(1.00-3.69)$ & 0.051 \\
\hline Upper & $1.08(0.75-1.56)$ & 0.688 & $1.32(0.81-2.16)$ & 0.266 & $0.60(0.20-1.82)$ & 0.365 & $0.29(0.04-2.32)$ & 0.242 \\
\hline Unknown & $1.15(0.94-1.39)$ & 0.169 & $1.31(1.00-1.72)$ & 0.046 & $1.70(0.97-2.97)$ & 0.064 & $1.90(0.97-3.73)$ & 0.061 \\
\hline \multicolumn{9}{|l|}{ Tumor size } \\
\hline$\leq 30 \mathrm{~mm}$ & $\mathrm{RF}$ & $\mathrm{RF}$ & $\mathrm{RF}$ & $\mathrm{RF}$ & $\mathrm{RF}$ & $\mathrm{RF}$ & $\mathrm{RF}$ & $\mathrm{RF}$ \\
\hline$>30 \mathrm{~mm}$ & $1.16(0.98-1.39)$ & 0.090 & $1.35(1.07-1.69)$ & 0.010 & $1.38(0.95-2.00)$ & 0.093 & $1.63(1.03-2.58)$ & 0.038 \\
\hline Unknown & $0.99(0.84-1.16)$ & 0.859 & $0.98(0.77-1.25)$ & 0.874 & $0.69(0.40-1.20)$ & 0.185 & $0.86(0.44-1.71)$ & 0.676 \\
\hline
\end{tabular}

OS, overall survival; CSS, cause-specific survival; SA, surgery alone; SA + CT, surgery plus perioperative chemotherapy; ELN, examined lymph node; SCC, squamous cell carcinoma; AC, adenocarcinoma; HR, hazard ratios; Cl, confidence interval. 
Table 3 OS and CSS risk shown between surgery alone and surgery + perioperative chemotherapy groups according to $T$ stage

\begin{tabular}{lcccccc}
\hline Group & No. of SA + CT & No. of SA & Multivariable HR $(95 \%$ Cl) of OS & $P$ & Multivariable HR (95\% Cl) of CSS & P \\
\hline T1 & 54 & 62 & $1.88(0.87-4.06)$ & 0.107 & $2.82(1.04-7.67)$ \\
T2 & 35 & 26 & $0.54(0.22-1.32)$ & 0.176 & $0.32(0.08-1.26)$ \\
T3 & 59 & 60 & $0.39(0.23-0.65)$ & $<0.001$ & $0.40(0.22-0.75)$ & 0.103 \\
\hline
\end{tabular}

Multivariate analysis adjusted by age of patients, race, gender, nuclear grade, histology, ELN count, tumor location and tumor size and treatment. OS, overall survival; CSS, cause-specific survival; SA, surgery alone; SA + CT, surgery plus perioperative chemotherapy; ELN, examined lymph node; HR, hazard ratios; $\mathrm{Cl}$, confidence interval.

group for T1 patients.

Chemotherapy is a common and effective adjunct therapy for EC, but there are some controversies. A multicenter phase III trial showed that perioperative chemotherapy significantly improved the OS and disease-free survival in patients with lower esophagus adenocarcinoma (9). Cunningham et al. reported that perioperative chemotherapy decreased tumor size and improved tumor stage $(10)$. However, other studies $(11,12)$ showed that preoperative chemotherapy did not improve outcomes of patients with EC. The limitation of these studies lies in the lack of stratified analysis of the results using the disease stage, which may be the reason for the inconsistent effect of chemotherapy on survival. In our study, SA + CT and the SA groups had a significant survival difference before PSM. However, no significant difference was found between the two groups after PSM. Similarly, Ando et al. (13) found that adjuvant chemotherapy could not improve the 5-year disease-free survival rate for lymph node negative patients. In this study, T3 patients could benefit from chemotherapy, whereas subgroup analysis showed that perioperative chemotherapy increases the risk of death in $\mathrm{T} 1$ patients; the CSS HR of T1 patients in the SA + CT group was approximately 2.8 compared with $\mathrm{T} 1$ patients in the SA group. The HR of CSS and OS in the SA group was nearly 2.5 for T3 patients, where the SA + CT group was taken as the reference. Therefore, this study suggests that SA is more effective for $\mathrm{T} 1$ stage patients, whereas patients at $\mathrm{T} 3$ stage should receive $\mathrm{SA}+\mathrm{CT}$.

Sato et al. (14) found that approximately $40 \%$ of patients at postoperatively confirmed pN0 stage had micrometastasis of lymph nodes. More micrometastasis was associated with a higher $\mathrm{T}$ stage. Another study showed that $\mathrm{pN} 0$ patients with lymph node micrometastasis contained $31.7 \%$ of all pN0 patients, and the survival time of these patients was similar to that of original $\mathrm{pN} 1$ patients (15). T3N0 patients may have potential lymph node micrometastasis, which may be related to effectiveness of perioperative chemotherapy in T3N0 patients. Perioperative chemotherapy may present the risk of toxicity compared with SA. The most common side effects of chemotherapy include general fatigue, leukopenia, anemia and stomatitis (16). Previous researches $(17,18)$ showed that neoadjuvant therapy could increase pneumonia, arrhythmia, and postoperative deaths. In this study, perioperative chemotherapy was correlated with poor CSS of T1 patients, which may be attributed to the adverse effects of chemotherapy. A previous study (19) found that $50 \%$ of cT2N0 patients were understated and recommended the use of neoadjuvant therapy. But a metaanalysis, including eight retrospective studies of 2,646 patients with T2N0 EC, showed no statistically significant difference in the 5 -year OS between the adjuvant therapy and SA groups (20). This was consistent with our results whereby perioperative chemotherapy showed no effect on OS and CSS of T2 EC. Further research should be carried out to explore effective treatment approaches for T2 EC.

There were several shortcomings in this study. There is no definitive protocol for chemotherapy, which makes it difficult to determine the accuracy of treatment strategies. Secondly, the data used in this study are from public databases, and the inclusion and exclusion criteria were not all clear. Besides, the study was retrospective and nonrandomized. Also, some variables such as resection margin, lymphovascular invasion, and gene mutations were not included in this database, which may also affect the survival of patients.

\section{Conclusions}

There is a significant survival benefit from perioperative chemotherapy for $\mathrm{T} 3 \mathrm{~N} 0$ patients. However, perioperative chemotherapy does not present survival benefit to T1-2 patients, and it is a risk factor for adverse clinical outcome of T1 patients. Unmeasurable confounding factors in the 

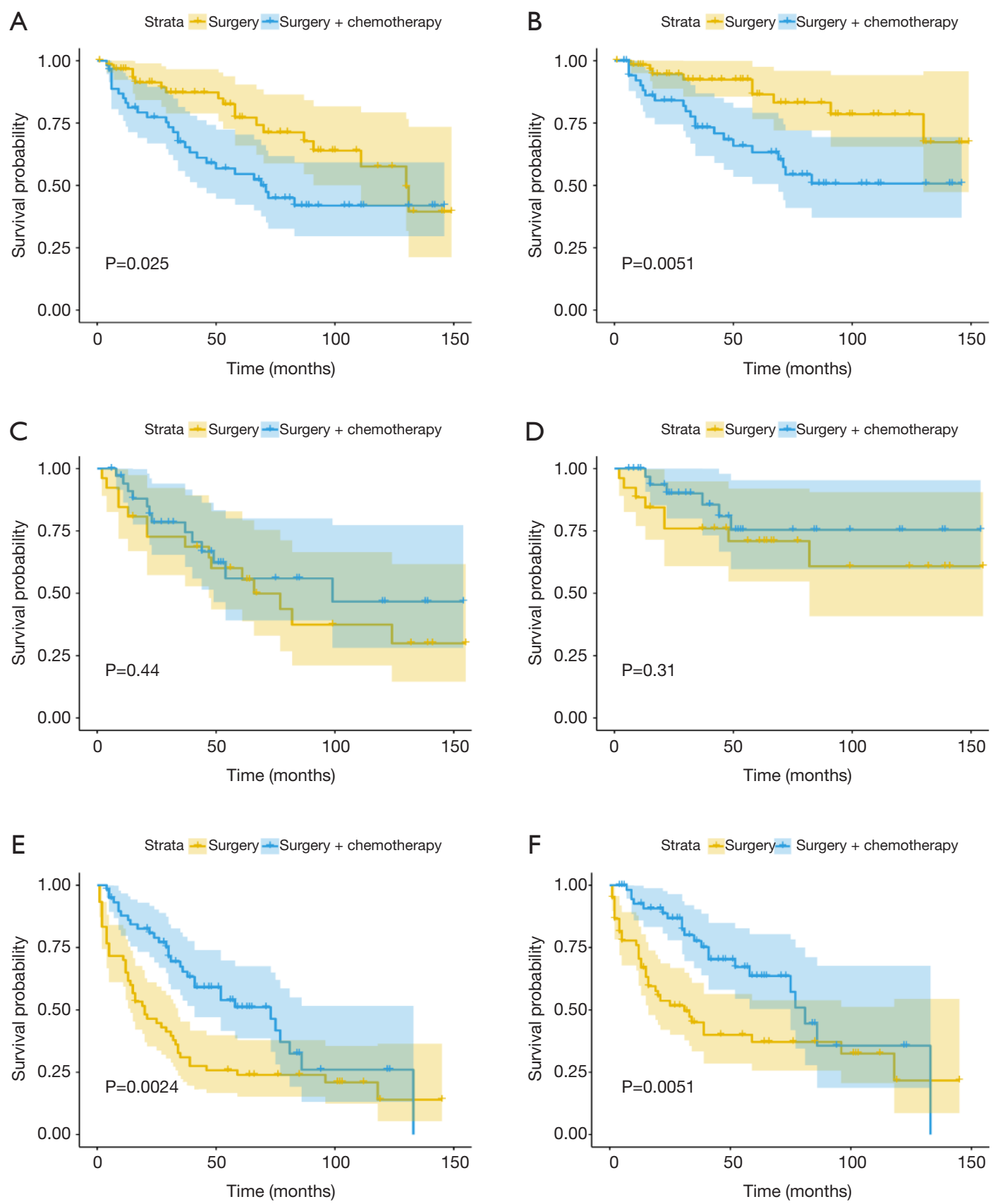

Figure 3 Overall survival and cause-specific survival of different $T$ stages after propensity score-matched. Overall survival between surgery alone and surgery + perioperative chemotherapy groups in pT1 subgroup (A), T2 subgroup (C) and T3 subgroup (E). Cause-specific survival comparisons between patients with surgery alone and surgery + perioperative chemotherapy in T1 subgroup (B), T2 subgroup (D) and T3 $\operatorname{subgroup}(\mathrm{F})$. 
SEER database limit these findings. Therefore, further studies should be carried out to validate the potential role of perioperative chemotherapy in lymph node-negative EC.

\section{Acknowledgments}

Funding: This study was supported by Health Bureau of Chongqing (Grant 20121015) and Chongqing Medical University Scholarship Fund for Development of Young Talents (No. XRJH201905).

\section{Footnote}

Reporting Checklist: The authors have completed the STROBE reporting checklist. Available at http://dx.doi. org/10.21037/jtd-20-2877

Conflicts of Interest: All authors have completed the ICMJE uniform disclosure form (available at http://dx.doi. org/10.21037/jtd-20-2877). The authors have no conflicts of interest to declare.

Ethical Statement: The authors are accountable for all aspects of the work in ensuring that questions related to the accuracy or integrity of any part of the work are appropriately investigated and resolved. The study was conducted in accordance with the Declaration of Helsinki (as revised in 2013). Requirement for informed consent was waived since the SEER database is publicly available and anonymous. This study was approved by the Ethics Committee of the First Affiliated Hospital of Chongqing Medical University, Chongqing, China as exempted research with no human subject involved.

Open Access Statement: This is an Open Access article distributed in accordance with the Creative Commons Attribution-NonCommercial-NoDerivs 4.0 International License (CC BY-NC-ND 4.0), which permits the noncommercial replication and distribution of the article with the strict proviso that no changes or edits are made and the original work is properly cited (including links to both the formal publication through the relevant DOI and the license). See: https://creativecommons.org/licenses/by-nc-nd/4.0/.

\section{References}

1. Liang H, Fan JH, Qiao YL. Epidemiology, etiology, and prevention of esophageal squamous cell carcinoma in
China. Cancer Biol Med 2017;14:33-41.

2. Sohda M, Kuwano H. Current status and future prospects for esophageal cancer treatment. Ann Thorac Cardiovasc Surg 2017;23:1-11.

3. Abbas G, Krasna M. Overview of esophageal cancer. Ann Cardiothorac Surg 2017;6:131-6.

4. Vosmik M. Technological advances in radiotherapy for esophageal cancer. World J Gastroenterol 2010;16:5555-64.

5. Won E, Ilson DH. Management of localized esophageal cancer in the older patient. Oncologist 2014;19:367-74.

6. Heroor A, Fujita H, Sueyoshi S, et al. Adjuvant chemotherapy after radical resection of squamous cell carcinoma in the thoracic esophagus: who benefits? Dig Surg 2003;20:229-35.

7. Wong AT, Shao M, Rineer J, et al The impact of adjuvant postoperative radiation therapy and chemotherapy on survival after esophagectomy for esophageal carcinoma. Ann Surg 2017;265:1146-51.

8. Gao SJ, Park HS, Corso CD, et al. Role of adjuvant treatment in esophageal cancer with incidental pathologic node positivity. Ann Thorac Surg 2017;104:267-74.

9. Ychou M, Boige V, Pignon JP, et al. Perioperative chemotherapy compared with surgery alone for resectable gastroesophageal adenocarcinoma: an FNCLCC and FFCD multicenter phase III trial. J Clin Oncol 2011;29:1715-21.

10. Cunningham D, Allum WH, Stenning SP, et al. Perioperative chemotherapy versus surgery alone for resectable gastroesophageal cancer. N Engl J Med 2006;355:11-20.

11. Kelsen DP, Ginsberg R, Pajak TF, et al. Chemotherapy followed by surgery compared with surgery alone for localized esophageal cancer. N Engl J Med 1998;339:1979-84.

12. Kelsen DP, Winter KA, Gunderson LL, et al. Longterm results of RTOG trial 8911 (USA Intergroup 113): a random assignment trial comparison of chemotherapy followed by surgery compared with surgery alone for esophageal cancer. J Clin Oncol 2007;25:3719-25.

13. Ando N, Iizuka T, Ide H, et al. Surgery plus chemotherapy compared with surgery alone for localized squamous cell carcinoma of the thoracic esophagus: a Japan Clinical 0ncology Group Study--JC0G9204. J Clin 0ncol 2003;21:4592-6.

14. Sato F, Shimada Y, Li Z, et al. Imamura M. Lymph node micrometastasis and prognosis in patients with oesophageal squamous cell carcinoma. Br J Surg 2001;88:426-32. 
15. Natsugoe S, Mueller J, Stein HJ, et al. Micrometastasis and tumor cell microinvolvement of lymph nodes from esophageal squamous cell carcinoma: frequency, associated tumor characteristics, and impact on prognosis. Cancer 1998;83:858-66.

16. Miyata H, Yano M, Yasuda T, et al. Randomized study of the clinical effects of $\omega-3$ fatty acid-containing enteral nutrition support during neoadjuvant chemotherapy on chemotherapy-related toxicity in patients with esophageal cancer. Nutrition 2017;33:204-10.

17. Bosch DJ, Muijs CT, Mul VE, et al. Impact of neoadjuvant chemoradiotherapy on postoperative course after curativeintent transthoracic esophagectomy in esophageal cancer

Cite this article as: Shao Y, Chen D, Ye L, Wang XM, Wu QC, Zhang C. Survival benefit of perioperative chemotherapy for T1-3N0M0 stage esophageal cancer: a SEER database analysis. J Thorac Dis 2021;13(2):995-1004. doi: 10.21037/jtd20-2877 patients. Ann Surg Oncol 2014;21:605-11.

18. Bosset JF, Gignoux M, Triboulet JP, et al.

Chemoradiotherapy followed by surgery compared with surgery alone in squamous-cell cancer of the esophagus. $\mathrm{N}$ Engl J Med 1997;337:161-7.

19. Zhang JQ, Hooker CM, Brock MV, et al. Neoadjuvant chemoradiation therapy is beneficial for clinical stage T2 N0 esophageal cancer patients due to inaccurate preoperative staging. Ann Thorac Surg 2012;93:429-35.

20. Lv HW, Xing WQ, Shen SN, Cheng JW. Induction therapy for clinical stage T2NOM0 esophageal cancer: A systematic review and meta-analysis. Medicine (Baltimore) 2018;97:e12651. 